\title{
Neurophysiological testing correlates with clinical examination according to fibre type involvement and severity in sensory neuropathy
}

\author{
J-P Lefaucheur, A Créange
}

J Neurol Neurosurg Psychiatry 2004;75:417-422. doi: 10.1136/jnnp.2003.019208

See end of article for authors' affiliations .....................

Correspondence to: Dr Jean-Pascal Lefaucheur, Service de Physiologie Explorations

Fonctionnelles, Hôpital Henri Mondor, 51 avenue de Lattre de Tassigny, 94010 Créteil cedex, France; jean-pascal.lefaucheur@ hmn.ap-hop-paris.fr

Received 20 May 2003 In revised form 28 July 2003 Accepted 6 August 2003

\begin{abstract}
Objective: To investigate a comprehensive battery of neurophysiological tests for objective evaluation of sensory neuropathies including fibre type involvement and severity, and to determine the relation between neurophysiological data and clinical examination.

Methods: 45 patients referred for sensory neuropathy were studied using a standardised clinical evaluation of large and small fibre symptoms and an original neurophysiological battery. Clinical evaluation included: assessment of tactile, vibratory, and pin sensation; tendon reflexes; toe position sense; ataxia score; pain level; and presence of trophic, vasomotor, or sudomotor abnormalities. The neurophysiological battery included: recording of large fibre and small fibre components of the sural sensory nerve action potential; somatosensory evoked cortical potentials and soleus $\mathrm{H}$ reflex following tibial nerve electrical stimulation; laser evoked potentials following $\mathrm{Nd}$ :YAG laser stimulation of the foot; and plantar sympathetic skin response to median nerve stimulation. Neuropathy was classified according to the predominantly affected fibre type, and a severity score was established based on clinical and neurophysiological abnormalities.

Results: On clinical examination there were 22 patients with large fibre sensory neuropathy (LFSN), 18 with mixed sensory neuropathy (MSN), and five with small fibre sensory neuropathy (SFSN). Neurophysiological classification identified 25 patients with LFSN, 13 with MSN, and seven with SFSN. Clinical and neurophysiological classifications and severity scores were correlated, whatever the type of neuropathy. Conclusions: The correlation between clinical examination and the results of an original neurophysiological test battery offers a comprehensive clinical and neurophysiological approach to the objective assessment of peripheral neuropathies according to fibre type involvement and overall severity.
\end{abstract}

diagnosis of peripheral neuropathy was obtained by standard clinical, biological, and electrophysiological investigations. Inclusion criteria were the presence of at least one of the following signs or symptoms involving the lower limbs:

- bilateral, symmetrical or asymmetrical numbness, paraesthesiae, pain, ataxia, areflexia, or dysautonomia;

- a chronic stable disorder over the three preceding months.

Patients with focal mononeuropathy, pure motor or motor predominant neuropathy, or neuropathy restricted to upper limbs were excluded. Patients with cognitive deterioration preventing an accurate understanding of tests, and those with associated central nervous system abnormalities were also excluded.

\section{Clinical classification}

Clinical assessment included a systematic evaluation of tendon reflexes, superficial and proprioceptive sensibility, pain, and trophic or vasomotor abnormalities. The clinical examination took about 10 minutes. Tendon reflexes, toe position sense (evaluated by the responses to 10 questions), vibratory skin sensation (measured by a $128 \mathrm{~Hz}$ tuning fork), tactile skin sensation, and pin skin sensation were evaluated according to the neurologic disability score (NDS) scale ${ }^{7}$ in the lower limbs (on the first toe, ankle, leg, and knee).

Abbreviations: LEP, laser evoked potential; LFSN, large fibre sensory neuropathy; MSN, mixed (large and small) sensory neuropathy; SEP, somatosensory evoked potential; SFSN, small fibre sensory neuropathy; smFC, small fibre component; SNAP, sensory nerve action potential; SSR, sympathetic skin response; VAS, visual analogue scale
Forty five patients with a sensory predominant peripheral neuropathy were included prospectively in the study. A 
Examination was considered as normal (0), decreased (1), or absent (2). The ataxia score was derived from the NobileOrazio score ${ }^{8}$ as follows: normal posture with closed eyes $(0)$; slight postural alteration with closed eyes (1); severe postural alteration with closed eyes (2); inability to stand with closed eyes (3). Dysautonomia was determined by the presence of trophic, vasomotor, or sudomotor abnormalities. Pain was evaluated as the spontaneous pain intensity on a $100 \mathrm{~mm}$ visual analogue scale (VAS) graduated from 0 (no pain) to 100 (worst possible pain).

Large fibre involvement was assumed in the following situations:

- loss of tactile or vibratory skin sensation in any part of a lower limb, assessing skin mechanoreceptors to touch, pressure, and vibration associated with A- $\beta$ (type II) sensory fibres;

- decreased or absent tendon reflexes, assessing muscle spindle receptors associated with $\mathrm{A}-\alpha$ (type I) sensory fibres;

- an ataxia score of $>1$ or alteration in the toe position sense, assessing joint proprioceptors also associated with A- $\alpha$ (type I) sensory fibres.

Small fibre involvement was assumed in the following situations:

- alteration of pin sensation in any part of a lower limb, assessing mechanonociceptors associated with A- $\delta$ (type III) sensory fibres;

- the presence of trophic, vasomotor, or sudomotor abnormalities;

- A VAS pain score of $>40$.

The last two variables assess the transmission of information mediated by lightly myelinated or unmyelinated autonomic or sensory fibres.

\section{Qualitative classification}

Each positive criterion was evaluated as 1 point, any unilateral abnormal result being sufficient to render the assessment of that entire criterion abnormal. Neuropathy was classified as LFSN when large fibre criteria were the majority, SFSN when small fibre criteria were the majority, and MSN when large and small fibre criteria were equal in number.

\section{Severity scoring}

The total number of positive criteria was used to evaluate the clinical severity of the neuropathy.

\section{Neurophysiological classification}

Eight neurophysiological tests were applied bilaterally. The neurophysiological examination took about 45 minutes. Clinical and neurophysiological testing was undertaken independently, and in each case the assessor was blinded to the findings of the other assessment. For all electrophysiological recordings we used either a Keypoint (Medtronic France, Boulogne-Billancourt, France) or a Phasis II (Esaote Biomedica, Florence, Italy) EMG-EP machine.

Sural nerve conduction was studied antidromically on both ankles, using subcutaneous needles, both for stimulation and for recording. The amplitude of the distal sensory nerve action potential (SNAP) of the both sural nerves was measured and averaged. Mean sural SNAP amplitudes of more than $15 \mu \mathrm{V}$ were considered normal. ${ }^{9}$ Subsequently, the small fibre component ( $\mathrm{smFC}$ ) of the sural SNAP was studied by averaging 1000 stimuli with an onset delay of $2 \mathrm{~ms} .{ }^{10}$ The presence of bilateral smFCs, whatever their amplitude, was considered normal.
Quantitative sensory testing was done on the dorsum of the foot using a VSA-3000/TSA-2001 device (Medoc, Ramat Yshai, Israel). The vibratory threshold and the thermal (warm and cold) sensory threshold (temperature threshold) were measured bilaterally using the method of limits. ${ }^{11}$ Vibration was tested at a constant frequency $(100 \mathrm{~Hz})$ but with increasing amplitude. Results obtained from the both feet were averaged to define the vibratory threshold. Normal values were less than $12 \mu \mathrm{m}$ for vibratory threshold at the feet. ${ }^{12}$ For thermal testing, temperature was increased (warm sensation) or decreased (cold sensation) at a linear rate of $1 \%$ $\mathrm{s}$ from a neutral temperature of $32^{\circ} \mathrm{C}$. The mean differential value between the temperature perceived as warm or cold and this neutral temperature was calculated from five trials. The overall mean differential value from bilateral warm and cold sensory testing then defined the mean temperature threshold. From published normative data, the upper normal limit for this value was estimated to be $12^{\circ} \mathrm{C} .^{11}$

The proprioceptive H reflexes were recorded over the soleus muscles following the stimulation of the tibial nerve at the popliteal fossa. The mean amplitude of the averaged right and left maximum $\mathrm{H}$ reflex equal to or greater than $1 \mathrm{mV}$ was considered normal, based on published data ${ }^{13}$ and our own laboratory reference. Plantar sympathetic skin responses (SSR) were recorded bilaterally following the electrical stimulation of the median nerve at the wrist. Three trials were done, using increasing stimulus intensities and random stimulation intervals to avoid habituation. The mean amplitude of the averaged right and left SSR equal to or greater than $1 \mathrm{mV}$ was considered normal. ${ }^{14}$

Somatosensory evoked potentials (SEP) were recorded at cortical level by means of subcutaneous needle electrodes placed in the scalp $(2 \mathrm{~cm}$ behind the vertex referred midfrontally) following repetitive electrical stimulation of the posterior tibial nerve at the ankle. Two sets of 250 stimuli were undertaken. The mean latency of the right and left P40 peaks was taken into account (upper limit of normal, 44 ms). ${ }^{15}$ Laser evoked potentials (LEP) were recorded at the vertex with extracephalic reference (linked earlobes) following Nd:YAG laser stimulation of the dorsum of the foot. ${ }^{16}$ Before any recording, the diameter of the illuminated area at the level of the skin was measured with a near-infrared sensitive paper and was maintained around $5 \mathrm{~mm}$. Laser pulses were delivered at a given energy of $300 \mathrm{~mJ}$, fixed for all patients, resulting in a mean energy density of $15 \mathrm{~mJ} / \mathrm{mm}^{2}$. Using this energy density, foot stimulation can, in our experience, elicit pinprick sensation and cortical LEP for all healthy subjects with a negative peak latency around 200 ms. ${ }^{16}$ Two sets of 20 stimuli were delivered with random intervals (ranging from 5 to 20 seconds) to avoid habituation, and were averaged for each side. The peak to peak amplitude of the vertex responses was measured. A response equal to or greater than $10 \mu \mathrm{V}$ in amplitude, averaged bilaterally, was considered normal.

\section{Qualitative classification}

SNAP and H reflex amplitude, vibratory threshold, and SEP latency were used to assess large diameter nerve fibres, while the presence of smFC and LEP, temperature threshold, and SSR amplitude were used to investigate small diameter nerve fibres. The neuropathy was classified as LFSN, SFSN, or MSN (equal number of large and small fibre abnormal parameters) according to the number of abnormal responses in each type of study.

\section{Severity scoring}

The total number of abnormal responses was used to obtain a neurophysiological severity score for the neuropathy. 
Statistical analysis

The relation between clinical and neurophysiological classification of the neuropathy was analysed for each type of neuropathy, and compared with the two other types using Fisher's exact test. The relation between clinical and neurophysiological severity scores of the neuropathies was analysed using Pearson's test.

\section{RESULTS}

\section{Patients}

Our series included 45 patients, 28 men (61\%) and 17 women (39\%). Their mean (SD) age at the time of the study was 64 (12) years (range 31 to 80 ). The neuropathy was determined by standard investigations as distal axonal polyneuropathy $(n=39)$ or polyradiculoneuropathy $(n=6)$. Electrophysiological classification was axonal neuropathy $(\mathrm{n}=36)$ or demyelinating neuropathy $(n=9)$. The cause of the neuropathy was metabolic $(n=18)$, infectious $(n=2)$, dysimmune/paraneoplastic $(n=10)$, or toxic $(n=4)$. Eleven cases were idiopathic.

\section{Clinical and neurophysiological examination}

The results of the clinical and neurophysiological examinations are given in tables 1 and 2 .
The following abnormal criteria were found in the clinical examination (table 1$)$ : tendon reflexes $(n=39)$, superficial and vibratory sensations $(n=32)$, pin sensation $(n=28)$, VAS score $(n=22)$, ataxia or toe position sense $(n=17)$, and trophic, vasomotor, or sudomotor abnormalities $(n=13)$. In the clinical examination overall, there were 22 patients with LFSN, 18 with MSN, and five with SFSN. The severity scores ranged from 1 (four patients) to 6 (three patients).

The following abnormal results were found in the neurophysiological examination (table 2 ): vibratory threshold $(\mathrm{n}=38), \mathrm{H}$ reflex amplitude $(\mathrm{n}=37)$, SEP latency $(\mathrm{n}=35), \quad$ SNAP amplitude $(\mathrm{n}=31), \quad$ smFC presence $(\mathrm{n}=29)$, LEP presence $(\mathrm{n}=25)$, temperature threshold $(\mathrm{n}=24)$, and SSR amplitude $(\mathrm{n}=22)$. In the neurophysiological classification overall, there were 25 patients with LFSN, 13 with MSN, and seven with SFSN. The severity scores ranged from 2 (two patients) to 8 (seven patients).

\section{Relation between clinical and neurophysiological evaluation}

The relation between the clinical and the neurological examination is outlined in table 3 .

On neurophysiological grounds, clinically defined LFSN were classified as LFSN $(n=17)$ or MSN $(n=8)$, but never as

Table 1 Individual results of the clinical evaluation

\begin{tabular}{|c|c|c|c|c|c|c|c|c|}
\hline Case No & $\begin{array}{l}\text { Tactile/vibratory } \\
\text { sensation }\end{array}$ & Tendon reflexes & $\begin{array}{l}\text { Posture/toe } \\
\text { position sense }\end{array}$ & $\begin{array}{l}\text { Pin prick } \\
\text { sensation }\end{array}$ & $\begin{array}{l}\text { Vasomotor/ } \\
\text { sudomotor trophic } \\
\text { abnormalities }\end{array}$ & Pain score & $\begin{array}{l}\text { Neuropathy } \\
\text { classification }\end{array}$ & $\begin{array}{l}\text { Neuropathy severity } \\
\text { score }\end{array}$ \\
\hline 1 & Abnormal & Abnormal & Normal & Normal & Absent & 7 & If & 3 \\
\hline 2 & Abnormal & Abnormal & Abnormal & Normal & Absent & 0.5 & If & 3 \\
\hline 3 & Abnormal & Abnormal & Normal & Abnormal & Absent & 0 & If & 3 \\
\hline 4 & Abnormal & Abnormal & Abnormal & Normal & Present & 3 & If & 4 \\
\hline 5 & Abnormal & Abnormal & Abnormal & Abnormal & Present & 4 & If & 5 \\
\hline 6 & Abnormal & Abnormal & Normal & Abnormal & Absent & 6 & $\mathrm{~m}$ & 4 \\
\hline 7 & Abnormal & Abnormal & Abnormal & Abnormal & Present & 5 & $\mathrm{~m}$ & 6 \\
\hline 8 & Abnormal & Abnormal & Abnormal & Abnormal & Absent & 6.5 & If & 5 \\
\hline 9 & Abnormal & Abnormal & Abnormal & Abnormal & Present & 4.5 & $\mathrm{~m}$ & 6 \\
\hline 10 & Abnormal & Abnormal & Normal & Abnormal & Absent & 1 & If & 3 \\
\hline 11 & Abnormal & Abnormal & Abnormal & Abnormal & Absent & 0 & If & 4 \\
\hline 12 & Abnormal & Abnormal & Normal & Abnormal & Absent & 5 & $\mathrm{~m}$ & 4 \\
\hline 13 & Normal & Normal & Normal & Normal & Absent & 5 & sf & 1 \\
\hline 14 & Normal & Abnormal & Normal & Normal & Absent & 5 & $\mathrm{~m}$ & 2 \\
\hline 15 & Abnormal & Abnormal & Abnormal & Abnormal & Absent & 1 & If & 4 \\
\hline 16 & Abnormal & Abnormal & Normal & Abnormal & Present & 0 & $\mathrm{~m}$ & 4 \\
\hline 17 & Abnormal & Abnormal & Normal & Abnormal & Present & 0 & $\mathrm{~m}$ & 4 \\
\hline 18 & Abnormal & Abnormal & Normal & Abnormal & Absent & 3.5 & If & 3 \\
\hline 19 & Abnormal & Abnormal & Abnormal & Abnormal & Present & 2 & If & 5 \\
\hline 20 & Normal & Abnormal & Normal & Abnormal & Absent & 3 & $\mathrm{~m}$ & 2 \\
\hline 21 & Normal & Abnormal & Normal & Normal & Absent & 1 & If & 1 \\
\hline 22 & Abnormal & Abnormal & Abnormal & Abnormal & Absent & 6 & If & 5 \\
\hline 23 & Abnormal & Abnormal & Normal & Abnormal & Absent & 6 & $\mathrm{~m}$ & 4 \\
\hline 24 & Normal & Normal & Normal & Normal & Present & 5.5 & sf & 2 \\
\hline 25 & Abnormal & Abnormal & Normal & Abnormal & Absent & 4.5 & $\mathrm{~m}$ & 4 \\
\hline 26 & Abnormal & Abnormal & Normal & Abnormal & Absent & 8 & $\mathrm{~m}$ & 4 \\
\hline 27 & Normal & Normal & Normal & Normal & Absent & 5.5 & sf & 1 \\
\hline 28 & Abnormal & Abnormal & Abnormal & Abnormal & Absent & 0 & If & 4 \\
\hline 29 & Abnormal & Normal & Abnormal & Abnormal & Present & 3 & $\mathrm{~m}$ & 4 \\
\hline 30 & Abnormal & Abnormal & Normal & Normal & Absent & 4.3 & If & 3 \\
\hline 31 & Abnormal & Abnormal & Abnormal & Normal & Absent & 0.5 & If & 3 \\
\hline 32 & Abnormal & Abnormal & Abnormal & Abnormal & Present & 0 & If & 5 \\
\hline 33 & Normal & Abnormal & Normal & Normal & Absent & 8.5 & $\mathrm{~m}$ & 2 \\
\hline 34 & Abnormal & Abnormal & Abnormal & Abnormal & Present & 8 & $\mathrm{~m}$ & 6 \\
\hline 35 & Abnormal & Abnormal & Normal & Abnormal & Absent & 2 & If & 3 \\
\hline 36 & Normal & Normal & Normal & Normal & Present & 6 & sf & 2 \\
\hline 37 & Abnormal & Normal & Normal & Abnormal & Absent & 3 & $\mathrm{~m}$ & 2 \\
\hline 38 & Normal & Abnormal & Abnormal & Normal & Absent & 5 & If & 3 \\
\hline 39 & Normal & Abnormal & Normal & Abnormal & Absent & 1 & $\mathrm{~m}$ & 2 \\
\hline 40 & Abnormal & Abnormal & Normal & Abnormal & Present & 5 & sf & 5 \\
\hline 41 & Normal & Abnormal & Normal & Normal & Absent & 5 & $\mathrm{~m}$ & 2 \\
\hline 42 & Normal & Abnormal & Normal & Normal & Absent & 8 & $\mathrm{~m}$ & 2 \\
\hline 43 & Normal & Abnormal & Normal & Normal & Absent & 3 & If & 1 \\
\hline 44 & Abnormal & Abnormal & Normal & Normal & Absent & 0 & If & 2 \\
\hline 45 & Abnormal & Abnormal & Abnormal & Abnormal & Absent & 3.5 & If & 4 \\
\hline
\end{tabular}


Table 2 Individual results of the neurophysiological evaluation

\begin{tabular}{|c|c|c|c|c|c|c|c|c|c|c|}
\hline $\begin{array}{l}\text { Case } \\
\text { No }\end{array}$ & $\begin{array}{l}\text { Sural SNAP } \\
\text { amplitude } \\
(\mu \mathrm{V})\end{array}$ & $\begin{array}{l}\text { H reflex } \\
\text { amplitude } \\
(\mathrm{mV})\end{array}$ & $\begin{array}{l}\text { Vibratory } \\
\text { threshold } \\
(\mu \mathrm{m})\end{array}$ & $\begin{array}{l}\text { P40 latency } \\
\text { (ms) }\end{array}$ & $\begin{array}{l}\text { Small fibre } \\
\text { component }\end{array}$ & $\begin{array}{l}\text { Plantar SSR } \\
\text { amplitude } \\
\text { (mV) }\end{array}$ & $\begin{array}{l}\text { Thermal } \\
\text { threshold } \\
\text { ( } \mathrm{C} \text { ) }\end{array}$ & $\begin{array}{l}\text { Foot LEP } \\
\text { amplitude } \\
(\mu \mathrm{V})\end{array}$ & $\begin{array}{l}\text { Neuropathy } \\
\text { classification }\end{array}$ & $\begin{array}{l}\text { Neuropathy } \\
\text { severity score }\end{array}$ \\
\hline 1 & 18 & 0.25 & 23.5 & 43 & Absent & 1.2 & 13.3 & 12 & $\mathrm{~m}$ & 4 \\
\hline 2 & 1.5 & 0 & 25 & 49.5 & Absent & 1.4 & 19.7 & 10 & If & 6 \\
\hline 3 & 13 & 0.1 & 25 & 47 & Present & 1.1 & 17.6 & 0 & If & 6 \\
\hline 4 & 1.2 & 0 & 25 & Absent & Absent & 0.4 & 18 & 0 & $\mathrm{~m}$ & 8 \\
\hline 5 & 33.5 & 0.35 & 24.5 & 46.9 & Present & 0.55 & 12.2 & 0 & $\mathrm{~m}$ & 6 \\
\hline 6 & 5.5 & 0.2 & 25 & 54.5 & Absent & 0.5 & 23 & 0 & $\mathrm{~m}$ & 8 \\
\hline 7 & 0 & 0.45 & 24 & Absent & Absent & 0.6 & 12.4 & 0 & $\mathrm{~m}$ & 8 \\
\hline 8 & 35 & 0 & 22 & Absent & Present & 8 & 13.6 & 13 & If & 4 \\
\hline 9 & 0 & 0.6 & 25 & Absent & Absent & 0.5 & 18 & 0 & $\mathrm{~m}$ & 8 \\
\hline 10 & 3.5 & 0 & 24 & Absent & Absent & 0 & 20 & 32 & If & 7 \\
\hline 11 & 4.5 & 0.1 & 25 & 57.6 & Absent & 0.5 & 9.9 & 44 & If & 6 \\
\hline 12 & 6 & 0 & 25 & 47.5 & Absent & 1.3 & 16 & 0 & If & 7 \\
\hline 13 & 17 & 1.2 & 19 & 43.5 & Present & 0.7 & 9.9 & 0 & sf & 3 \\
\hline 14 & 18 & 0.8 & 4.5 & 43.5 & Absent & 3.7 & 8.2 & 6 & $\mathrm{~m}$ & 2 \\
\hline 15 & 3.5 & 0.5 & 23 & 51.4 & Absent & 1.1 & 19.7 & 0 & If & 7 \\
\hline 16 & 4 & 0.05 & 18.5 & 52.9 & Absent & 2.55 & 11.7 & 0 & If & 6 \\
\hline 17 & 0 & 0 & 25 & 58 & Absent & 1.45 & 10.3 & 0 & If & 6 \\
\hline 18 & 9 & 0.3 & 22.5 & 49.7 & Present & 1.2 & 25 & 12.5 & If & 5 \\
\hline 19 & 11 & 0.1 & 22.5 & 55.5 & Absent & 1.1 & 16.5 & 11 & If & 6 \\
\hline 20 & 6.5 & 0.25 & 25 & Absent & Absent & 0.8 & 19 & 0 & $\mathrm{~m}$ & 8 \\
\hline 21 & 16 & 0.2 & 21 & 40.1 & Present & 0.9 & 9.4 & 0 & $\mathrm{~m}$ & 4 \\
\hline 22 & 11 & 1.3 & 19 & 45.6 & Present & 1.1 & 18.8 & 10 & If & 4 \\
\hline 23 & 2.5 & 0.1 & 24.5 & 60 & Absent & 2.7 & 12.5 & 0 & If & 7 \\
\hline 24 & 32 & 1.1 & 10.5 & 43.8 & Absent & 0.5 & 9.5 & 0 & sf & 3 \\
\hline 25 & 0 & 0.05 & 15.2 & Absent & Absent & 0.85 & 6.9 & 13 & If & 6 \\
\hline 26 & 1 & 0.1 & 24.5 & 55 & Absent & 0.4 & 16.6 & 0 & $\mathrm{~m}$ & 8 \\
\hline 27 & 17 & 0.15 & 6.6 & 47.5 & Absent & 5.7 & 6.7 & 0 & $\mathrm{~m}$ & 4 \\
\hline 28 & 0 & 0 & 25 & Absent & Absent & 0 & 13.7 & 0 & $\mathrm{~m}$ & 8 \\
\hline 29 & 6 & 1 & 25 & 50 & Absent & 0.4 & 13.7 & 0 & sf & 7 \\
\hline 30 & 9.5 & 1 & 23.2 & 51.6 & Present & 0.8 & 9.6 & 0 & If & 5 \\
\hline 31 & 3.5 & 0.3 & 25 & 49.3 & Present & 1.4 & 8.1 & 13 & If & 4 \\
\hline 32 & 10 & 0.05 & 21 & Absent & Present & 2.9 & 9.8 & 15.5 & If & 4 \\
\hline 33 & 13.5 & 0.1 & 14.4 & 43.5 & Absent & 1.8 & 23.9 & 0 & $\mathrm{~m}$ & 6 \\
\hline 34 & 13 & 0.3 & 25 & 44.2 & Absent & 0.2 & 19.1 & 12 & If & 7 \\
\hline 35 & 12 & 0.3 & 22.2 & 51 & Present & 2.5 & 3.1 & 13 & If & 4 \\
\hline 36 & 15.5 & 2.8 & 11.3 & 49.7 & Absent & 0.8 & 8.5 & 10 & sf & 3 \\
\hline 37 & 21 & 0.35 & 25 & 51.8 & Present & 0.85 & 9.3 & 12.5 & If & 4 \\
\hline 38 & 3.5 & 0.2 & 17 & 53.5 & Absent & 1.5 & 9.8 & 10 & If & 5 \\
\hline 39 & 13 & 1.7 & 20 & Absent & Absent & 1.8 & 8.4 & 13 & If & 4 \\
\hline 40 & 7 & 1 & 11 & 53 & Absent & 0 & 5.1 & 0 & sf & 5 \\
\hline 41 & 42 & 0.3 & 1.5 & 35.4 & Absent & 0.5 & 9.7 & 0 & sf & 4 \\
\hline 42 & 22 & 0.05 & 7 & 43.4 & Present & 0 & 10 & 0 & sf & 3 \\
\hline 43 & 16 & 0.3 & 15.5 & 43.3 & Present & 1 & 12.6 & 10 & If & 3 \\
\hline 44 & 5 & 0 & 25 & 62.6 & Present & 2.5 & 15.4 & 0 & If & 6 \\
\hline 45 & 23 & 0.7 & 15.5 & 40.7 & Present & 5 & 6.6 & 36 & If & 2 \\
\hline
\end{tabular}

SFSN; clinically defined SFSN were classified as SFSN $(\mathrm{n}=4)$ or MSN $(\mathrm{n}=3)$, but never as LFSN. On clinical grounds, neurophysiologically defined LFSN were classified as LFSN $(n=17)$ or MSN $(n=5)$, but never as SFSN; neurophysiologically defined SFSN were classified as SFSN $(\mathrm{n}=4)$ or MSN $(\mathrm{n}=1)$, but never as LFSN. Thus qualitative clinical and neurophysiological classifications were correlated for both LFSN and SFSN ( $p=0.007$ and 0.001 , respectively; Fisher's exact test), but not for MSN $(p=0.317)$. Clinical and electrophysiological severity scores were also correlated $(r=0.52 ; \mathrm{p}=0.0003 ;$ Pearson's test $)$.

Table 3 Numbers of patients in clinical and neurophysiological classification determined by fibre type involvement

\begin{tabular}{lllll}
\hline & \multicolumn{3}{l}{ Neurophysiological classification } \\
\cline { 3 - 5 } & & SFSN & MSN & LFSN \\
\hline Clinical & SFSN & 4 & 1 & 0 \\
classification & MSN & 3 & 7 & 8 \\
& LFSN & 0 & 5 & 17
\end{tabular}

LFSN, large fibre sensory neuropathy; MSN, mixed sensory neuropathy; SFSN, small fibre sensory neuropathy.

\section{DISCUSSION}

Recent studies have attempted to classify sensory neuropathies on the basis of the affected fibre population, particularly the description of sensory neuropathies in relation to selective lesions of small nerve fibre endings. ${ }^{17}$ This idiopathic distal small fibre neuropathy leads to disabling neuropathic symptoms, such as burning feet sensation, without any abnormalities on classical nerve conduction studies or nerve biopsy. At present, detection of SFSN is based on epidermal nerve fibre density measurement in skin biopsies, ${ }^{18}$ on quantitative thermal sensory testing, ${ }^{19}$ or on autonomic nervous system testing. ${ }^{20}$ In the present study, we propose an original evaluation test battery, including several different tests to investigate the various components of the sensory nerve, though the criteria-both clinical and neurophysiological-were defined arbitrarily without any system of weighting. Nevertheless, this strategy revealed a correlation between neurophysiological and clinical evaluations for both qualitative classification and severity scoring.

On clinical grounds, several approaches have been introduced to assess sensory deficits in the polyneuropathies. ${ }^{24} 7$ Although different scores have been validated, there are caveats that limit their use in clinical practice. First, these scores were not designed to classify neuropathies according 
to the predominance of the fibre type component; they were designed to evaluate all types of neuropathy on the basis of various motor, sensory, or autonomic symptoms. ${ }^{3} 6$ For instance, the neuropathy symptom profile was developed as an epidemiological tool and a screening questionnaire rather than for objective evaluation. ${ }^{4}$ More recently, a total neuropathy score was developed to focus on length dependent distal polyneuropathies. ${ }^{2}$ It has the major advantage of being easy to do, but it combines motor and sensory evaluations and clinical and objective variables. In addition, none of these composite scores includes any rating of spontaneous pain intensity on a visual analogue scale or an ataxia score, though these criteria are of interest for investigating small or large fibre components in peripheral sensory neuropathies. ${ }^{81}$

In the present study, we undertook a clinical evaluation that combined various non-redundant items of previously validated scores with pain ratings and an ataxia scale. It has been suggested that, from a clinical point of view, distinguishing the type of functional involvement is a help in guiding paraclinical investigations. Our results support this view by providing correlations between clinical and neurophysiological assessments. The present composite clinical evaluation could therefore be suitable for diagnosis, particularly in patients with only subjective signs and a normal neurological examination. For instance, in the present series, two patients presented with one purely subjective clinical sign (a VAS score of $>40 \mathrm{~mm}$ ) but with objective neurophysiological signs of neuropathy.

On neurophysiological grounds, various composite scores of nerve conduction parameters have been described in order to define abnormal results and to assess the severity of a neuropathy. ${ }^{22-24}$ Recently, Dyck and coworkers introduced composite scores of attributes of nerve conduction which were expressed as centiles and normal deviates, based on the study of these indices in large normative populations with correction for age, sex, and body mass index. ${ }^{25}$ However, the development of valid methods such as these is limited by the availability of large normative databases and the use of sophisticated statistical techniques. With the same objective, we developed a software tool, Diagnostica ${ }^{\circledR}$, to provide a single index representative of the overall electrophysiological values. This has been applied in several neuropathic conditions. ${ }^{26-28}$ Standardised electrophysiological data can also be used to delineate the pathophysiological mechanisms of a neuropathy-for example, axonal versus demyelinating. ${ }^{29}$ However, all these quantitative approaches are global and based on routine nerve conduction (large fibre) parameters, and have never been concerned with investigating the fibre type.

To define sensory neuropathy according to the affected nerve fibre population requires the use of unusual neurophysiological methods, particularly for small diameter nerve fibre assessment. Only SNAP measurements are commonly undertaken in routine neurophysiological examination of sensory neuropathies. In contrast, our present study was based on eight different tests: SNAP and H reflex amplitude, vibratory threshold, and SEP latency to investigate the large fibres, and smFC and LEP presence, temperature threshold, and SSR amplitude to investigate the small fibres. None of the results obtained by using these eight tests was redundant, thus justifying the use of the whole battery. Each of these methods had advantages and disadvantages, some of which we will discuss.

The smFC of the sural sensory nerve action potential has rarely been studied, though it was described a long time ago. $^{30}$ The main component of the sensory nerve action potential is related to the response of large diameter A- $\beta$ sensory nerve fibres (larger than $9 \mu \mathrm{m}$ in diameter), which represent only $30 \%$ of all myelinated fibres in the sural nerve. ${ }^{31}$ By means of near-nerve needle recording and averaging methods, some small, later occurring components can be observed. The first late component corresponds to fibres with conduction velocities in the range of 10 to 20 $\mathrm{m} / \mathrm{s}$ - that is, the thinly myelinated A- $\delta$ type of nerve fibres. ${ }^{10}$ This test was the most sensitive one for small fibre function, but it was felt to be relatively invasive by the patients because of the need for needle recording and for a large number of averaged stimuli. In addition, the smFC appeared to have an all or none response, and determination of conduction slowing or analysis of ultra-late components was highly speculative.

This latter drawback can also be applied to LEP, which mostly show an all or none response. LEP have rarely been used to investigate patients suffering from peripheral neuropathies, ${ }^{62} 33$ but they were found to correlate with pathological findings in the peripheral nerve. ${ }^{34}$ In fact, LEP explore concomitantly the peripheral conduction of A- $\delta$ nerve fibres and central conduction in the spinothalamic tract. In contrast to electrical SEP, it is impossible to record spinal responses and to distinguish between peripheral and central conduction time. This may represent a limit for the application of this technique. The same limit characterises quantitative sensory testing. Nevertheless, the latter offers the advantage of providing quantified values though it requires the patient's cooperation.

In this study we compared the respective value of various neurophysiological approaches to investigate similar nerve fibre pathways. We used nerve action potential recordings (SNAP, smFC), cortical evoked potential recording (SEP, LEP), sensory threshold measurements (vibratory thresholds, temperature thresholds), and reflexes ( $\mathrm{H}$ reflexes, SSR). For instance, similar results for LEP and temperature thresholds could be expected, but we did not find that the tests were redundant, as already reported. ${ }^{65}$ LEP depend on nerve conduction principles, while temperature threshold explores a nervous system function. With respect to SEP and vibratory thresholds, the vibratory threshold was more often altered, resulting in part from the fact that it explores a more distal territory than SEP.

Two types of reflex have been included in the battery. The SSR explores distal autonomic nerve fibres, but its variability limits its application in longitudinal studies. ${ }^{36}$ In fact, SSR amplitude was the neurophysiological parameter that was least often abnormal in the present study, though it remains an interesting complementary test in the investigation of length dependent neuropathies-as has been reported in diabetic and uraemic neuropathies. ${ }^{37-39}$ It is better established that patients with diabetic or uraemic neuropathy show early subclinical abnormalities of the soleus $\mathrm{H}$ reflex. ${ }^{40-42}$ The high rate of abnormality in this test was confirmed in the present study.

Such a comprehensive clinical and neurophysiological strategy for assessing fibre type involvement in sensory neuropathies has not been reported before. This approach should be of interest in improving neuropathy diagnoses in clinical practice. Our neurophysiological battery covered the spectrum of fibre types and was not limited to large fibres; if the results are confirmed, the method could be of value in clinical trials. It could be useful in the objective longitudinal assessment of sensory neuropathies, though the present study was not designed to address the question of follow up investigations. Various non-classical neurophysiological methods can supply useful information, complementary to the standard electrophysiological tests, particularly for the investigation of small diameter nerve fibres. These neurophysiological techniques can be applied in the form of a battery of sensitive, reproducible, specific, and non-invasive 
tests required for the objective assessment of peripheral neuropathies.

\section{ACKNOWLEDGEMENTS}

This work was presented in part at the annual meeting of the American Academy of Neurology, Honolulu, April 2003.

\section{Authors' affiliations}

J-P Lefaucheur, A Créange, INSERM E00.11, Hôpital Henri Mondor, Assistance Publique - Hôpitaux de Paris, Créteil, France

Competing interests: none declared

\section{REFERENCES}

1 Dyck PJ, Bushek W, Spring EM, et al. Vibratory and cooling detection thresholds compared with other tests in diagnosing and staging diabetic neuropathy. Diabetes Care 1987; 10:432-40.

2 Cornblath DR, Chaudhry V, Carter K, et al. Total neuropathy score: validation and reliability study. Neurology 1999;53:1660-4.

3 Dyck PJ, Sherman WR, Hallcher LM, et al. Human diabetic endoneurial sorbitol, fructose and myo-inositol related to sural nerve morphometry. Ann Neurol 1980;8:590-6.

4 Dyck PJ, Karnes J, O'Brien PC, et al. Neuropathy symptom profile in health, motor neuron disease, diabetic neuropathy, and amyloidosis. Neurology 1986;36:1300-8.

5 Santiago S, Ferrer T, Espinosa ML. Neurophysiological studies of thin myelinated (A delta) and unmyelinated (C) fibers: application to peripheral neuropathies. Neurophysiol Clin 2000;30:27-42

6 Lefaucheur JP, Brusa A, Créange A, et al. Clinical application of laser evoked potentials using the Nd:YAG laser. Neurophysiol Clin 2002;32:91-8.

7 Dyck PJ, Kratz KM, Lehman KA, et al. The Rochester diabetic neuropathy study: design, criteria for types of neuropathy, selection bias, and reproducibility of neuropathic tests. Neurology 1991;41:799-807.

8 Nobile-Orazio E, Baldini L, Barbieri S, et al. Treatment of patients with neuropathy and anti-MAG IgM M-proteins. Ann Neurol 1988;24:93-7.

9 Truong XT, Russo Fl, Vagi I, et al. Conduction velocity in the proximal sural nerve. Arch Phys Med Rehabil 1979;60:304-8

10 Shefner JM, Buchthal F, Krarup C. Slowly conducting myelinated fibers in peripheral neuropathy. Muscle Nerve 1991;14:534-42.

11 Yarnitsky D, Sprecher E. Thermal testing: normative data and repeatability for various test algorithms. J Neurol Sci 1994; 125:39-45.

12 Goldberg JM, Lindblom U. Standardised method of determining vibratory perception thresholds for diagnosis and screening in neurological investigation. J Neurol Neurosurg Psychiatry 1979;42:793-803.

13 Maryniak O, Yaworski R. H-reflex: optimum location of recording electrodes. Arch Phys Med Rehabil 1987;68:798-802.

14 Lefaucheur JP, Becquemin JP, Brugières $P$, et al. Assessment of sympathetic nerve activity in the practice of lumbar sympatholysis: interest of sympathetic skin responses. J Auton Nerv Syst 1996;60:56-60.

15 Pelosi L, Cracco JB, Cracco RQ, et al. Comparison of scalp distribution of short latency somatosensory evoked potentials (SSEPs) to stimulation of different nerves in the lower extremity. Electroencephalogr Clin Neurophysiol 1988:71:422-8.

16 Lefaucheur JP, Debray S, Jarry G. Laser evoked potentials with Nd:YAG laser pulses. Muscle Nerve 2001;24:496-501.

17 Holland NR, Crawford TO, Hauer P, et al. Small-fiber sensory neuropathies: clinical course and neuropathology of idiopathic cases. Ann Neurol 1998:44:47-59.

18 Periquet MI, Novak V, Collins MP, et al. Painful sensory neuropathy: prospective evaluation using skin biopsy. Neurology 1999;53:1641-7.

19 Navarro X, Kennedy WR. Evaluation of thermal and pain sensitivity in type I diabetic patients. J Neurol Neurosurg Psychiatry 1991;54:60-4.
20 Stewart JD, Low PA, Fealey RD. Distal small fiber neuropathy: results of tests of sweating and autonomic cardiovascular reflexes. Muscle Nerve 1992;15:661-5

21 Bouhassira D, Attal N, Willer JC, et al. Painful and painless peripheral sensory neuropathies due to HIV infection: a comparison using quantitative sensory evaluation. Pain 1999;80:265-72.

22 Solders G, Andersson T, Borin Y, et al. Electroneurography index: a standardized neurophysiological method to assess peripheral nerve function in patients with polyneuropathy. Muscle Nerve 1993;16:941-6.

23 Dyck PJ, Davies JL, Litchy WJ, et al. Longitudinal assessment of diabetic polyneuropathy using a composite score in the Rochester diabetic neuropathy study cohort. Neurology 1997;49:229-39.

24 Robinson LR, Micklesen PJ, Wang L. Strategies for analyzing nerve conduction data: superiority of a summary index over single tests. Muscle Nerve 1998;21:1166-71.

25 Dyck PJ, Litchy WJ, Daube JR, et al. Individual attributes versus composite scores of nerve conduction abnormality: sensitivity, reproducibility, and concordance with impairment. Muscle Nerve 2003;27:202-10.

26 Lefaucheur JP, Authier FJ, Verroust J, et al. Zidovudine and human immunodeficiency virus-associated peripheral neuropathies: low intake in patients with mononeuropathy multiplex and no evidence for neurotoxicity. Muscle Nerve 1997; 20:106-9.

27 Feki I Lefaucheur JP Correlation between nerve conduction studies and clinical scores in diabetic neuropathy. Muscle Nerve 2001;24:555-8.

28 Sharshar T, Durand MC, Lefaucheur JP, et al. MMP-9 correlates with electrophysiologic abnormalities in Guillain-Barré syndrome. Neurology 2002;59:1649-51

29 Hadden R, Cornblath D, Hughes R, et al. Electrophysiological classification of Guillain-Barré syndrome: clinical associations and outcome. Ann Neurol 1998;44:780-8.

30 Behse F, Buchthal F. Normal sensory conduction in the nerves of the leg in man. J Neurol Neurosurg Psychiatry 1971;34:404-14.

31 Buchthal F, Rosenfalck A, Behse F. Sensory potentials of normal and diseased nerves. In: Dyck PJ, Thomas PK, Lambert EH, Bunge R, eds. Peripheral neuropathy. Philadelphia: WB Saunders, 1984:981-1015.

32 Kakigi R, Shibasaki H, Ikeda T, et al. Pain-related somatosensory evoked potentials following $\mathrm{CO}_{2}$ laser stimulation in peripheral neuropathies. Acta Neurol Scand 1992;85:347-52.

33 Agostino R, Cruccu G, Romaniello A, et al. Dysfunction of small myelinated afferents in diabetic polyneuropathy, as assessed by laser evoked potentials. Clin Neurophysiol 2000;111:270-6.

34 Kakigi R, Shibasaki $\mathrm{H}$, Tanaka K, et al. $\mathrm{CO}_{2}$ laser-induced pain-related somatosensory evoked potentials in peripheral neuropathies: correlation between electrophysiological and histopathological findings. Muscle Nerve 1991;14:441-50.

35 Bromm B, Frieling A, Lankers J. Laser-evoked potentials in patients with dissociated loss of pain and temperature sensibility. Electroenceph Clin Neurophysiol 1991;80:284-91.

36 Hoeldtke RD, Davis KM, Hsieh PB, et al. Autonomic surface potential analysis: assessment of reproducibility and sensitivity. Muscle Nerve 1992;15:926-31.

37 Soliven B, Maselli R, Jaspan J, et al. Sympathetic skin response in diabetic neuropathy. Muscle Nerve 1987;10:711-16.

38 Niakan E, Harati Y. Sympathetic skin response in diabetic peripheral neuropathy. Muscle Nerve 1988;11:261-4

39 Wang SJ, Liao KK, Liou HH, et al. Sympathetic skin response and R-R interval variation in chronic uremic patients. Muscle Nerve 1994;17:411-18.

40 Bertelsman FW, Heimans JJ, van Rooy JC, et al. Comparison of Hoffmann reflex with quantitative assessment of cutaneous sensation in diabetic neuropathy. Acta Neurol Scand 1986;74:121-7.

41 Henriksen PH, Oey PL, Wieneke GH, et al. Subclinical diabetic polyneuropathy: early detection of involvement of different nerve fibre types. J Neurol Neurosurg Psychiatry 1993;56:509-14.

42 De Weerd AW, Nihom J, Rozeman CA, et al. H reflexes as a measure for uremic polyneuropathy. A longitudinal study in patients treated with dialysis or renal transplantation. Electroencephalogr Clin Neurophysiol 1994;93:276-80. 